\title{
The Anesthesiologist as a Medical Teacher
}

The primary responsibility of the anesthesiologist is patient care. The training of students is an equally crucial and demanding task. Most of us as clinicians have no formal training in teaching. The medical education units attached to the medical colleges try to fill this lacuna. The importance of such training has increased as society needs selfless dedicated doctors. The respect and trust that doctors have traditionally commanded in our society is waning. The insensitive behavior of caregivers is a matter of grave concern. It comes as no surprise that the spotlight on medical education is now intense. All of us are familiar with cognitive, psychomotor, and affective domains. If accumulation of knowledge is the mind and acquisition of technical skills the body, then medical ethics has to be the soul of medical education. The recognition that the attitude of the caregiver can affect the doctor-patient relationship tremendously has prompted the inclusion of an Attitudes and Communications (ATCOM) module in medical education. The realization that we as teachers are expected to impart not only basic clinical and communication skills but also sound moral reasoning and ethical judgment is daunting. Promotion of a healthy attitude requires the creation of an environment where the seniors inspire by example. This inspiring environment is required not only at the workplace but also in society as a whole. This implies a collective determination rather than individual effort. It is debatable whether an unethical society can expect to produce ethical doctors.

The conflict between clinical duties and academic schedules is a familiar one. Limited time has rendered "compressed," exam-oriented courses attractive. Our speciality is a stressful one. The trainees also learn how to handle stress from their teachers.

As complex technology is incorporated in anesthesia practice, the teacher has to necessarily acquire skills that were nonessential just three decades ago. Use of ultrasound, echocardiography, and application of digital simulation are just a few examples of such skills. The anesthetist is presently confronted with ethical issues related to brain death, organ transplant, and withdrawal of life support systems. The future is likely to add to this list.

It is relatively simple to present facts but not so simple to transfer the wisdom gained through experience. The art and craft of our speciality needs to be transmitted in a manner that encourages the acquisition of not only psychomotor skills but also the ability to handle complex ethical issues with sensitivity. This is a challenge for us as teachers and we have to accept it.

\section{Suggested Reading}

1. Bhuiyan PS, Rege NN, Supe A. The Art of Teaching Medical Students (3rd ed), Elsevier, Mumbai, Maharashtra, India, 2015. 